\title{
The variability of the optical spectra of three type 1 AGNs
}

D. Ilić*i

Department of Astronomy, Faculty of Mathematics, University of Belgrade, Republic of Serbia E-mail: dilicematf.bq.ac.rs

\section{L. Č. Popović}

Astronomical Observatory of Belgrade, Republic of Serbia

Department of Astronomy, Faculty of Mathematics, University of Belgrade, Republic of Serbia E-mail: Lpopoviceaob.rs

\section{A. I. Shapovalova}

Special Astrophysical Observatory of the Russian AS, Russia

E-mail: ashapdsao.ru

\section{A. N. Burenkov}

Special Astrophysical Observatory of the Russian AS, Russia

E-mail: bandsao.ru

\section{H. Chavushyan}

Instituto Nacional de Astrofisica, Óptica y Electrónica, México

E-mail: vahrameinaoep.my

\section{A. Kovačević}

Department of Astronomy, Faculty of Mathematics, University of Belgrade, Republic of Serbia E-mail: andjelkadmatf.bo.ac.rs

\section{W. Kollatscnhy}

Institut fuer Astrophysik, Germany

E-mail: wkollateastro.physik.uni-goettingen.de

We present the long-term optical monitoring campaign of a sample of type 1 active galactic nuclei (AGN) with different spectral properties. We give the analysis of the continuum and emission line properties with the aim to study the physics and kinematics of the broad-line region (BLR), which parameters are used to estimate the black hole mass in AGN. Especially, we present the results from the monitoring of the NLSy 1 galaxy Ark 564 with strong and variable Fe II emission, comparing them with our findings for the double-peaked line radio galaxy 3C390.3, and Seyfert 1.5 NGC 4151 with highly variable broad emission-line profiles.

Nuclei of Seyfert galaxies and QSOs - Central engine \& conditions of star formation,

November 6-8, 2012

Max-Planck-Insitut fuer Radioastronomie (MPIfR), Bonn, Germany

\footnotetext{
* Speaker.

${ }^{\dagger}$ This work is supported by the Ministry of Education and Science of R. Serbia through project 176001, and by RFBR (grants 12-02-01237a, 12-02-00857a).
} 


\section{Introduction}

The variability of astrophysical objects is an important tool to study in more details and better constrain their physical and kinematical properties. Active galactic nuclei (AGN) are objects that often show a variability across the whole electromagnetic spectrum, on both short and long timescales, as e.g. the continuum flux variability from the $\gamma$-ray to radio band, broad-line flux and profile variability, etc. Here we present a study of the spectral properties of type 1 AGN, objects with broad-emission lines (BELs) in there spectra, which properties can be used to estimate the mass of the super-massive black hole (SMBH).

BELs originate relatively close to the central power source, i.e. in the so called broad line region (BLR). The BLR is in the strong interaction with the radiation field produced by the central engine and is under the influence of the gravitational field of the SMBH, thus can be used to determine the SMBH mass using the virial theorem (see e.g. [焑]). From the long-term optical spectral monitoring of AGN, a time lag in the response of the BEL flux relative to flux changes in the continuum has been found (see e.g. [펴, [1, [2]). Assuming that the photoionization by the continuum source is the main heating mechanism, this lag gives us the size of the BLR, needed for the virial estimates of the SMBH mass. Nowadays, we use reverberation mapping techniques (see e.g. [四, 团], etc.) and their results and application (see e.g. [9, 피, 口, []], etc.) for estimates of the BLR size and SMBH mass. However, the accurate estimates of the lag and SMBH mass depends on the size, inclination, geometry, and physical conditions of the BLR, thus it is still important to study in details the BLR structure, and for this the analysis of the BELs properties (flux, profiles, widths, etc.) is needed.

We have carried out a long-term spectral optical monitoring campaign of a sample of type 1 AGN in Russia and Mexico. The main aim of the campaign is to monitor the variability of BELs in the optical domain (spectral ranges near $\mathrm{H} \alpha$ and $\mathrm{H} \beta$ ) and continuum, and investigate the structure (physics and kinematics) of the BLR. Here we describe some results about the BLR complex structure in the light of the reverberation mapping analysis, photoionization models, and SMBH mass estimates. In particular, we describe the results of Ark 564, a special case of an AGN class called narrow-line Seyfert 1 (NLS1) galaxies, 3C390.3, a double-peaked line (DPL) radio galaxy, and NGC 4151, a Seyfert 1.5 with highly variable broad emission-line profiles.

\section{Long-term optical monitoring campaign}

The long-term spectral optical monitoring campaign consisted from coordinated international observations of a sample of type 1 AGN, constantly carried out at four telescopes of Russia and Mexico during the period 1996-2012. The used instruments are: the 6-m and 1-m telescopes of SAO RAS, the 2.1-m telescope of INAOE (Cananea, México), and the $2.1 \mathrm{~m}$ telescope of OAN-SPM (Baja California, México). High quality spectra $(\mathrm{S} / \mathrm{N}>50)$ around the $\mathrm{H} \alpha$ and $\mathrm{H} \beta$

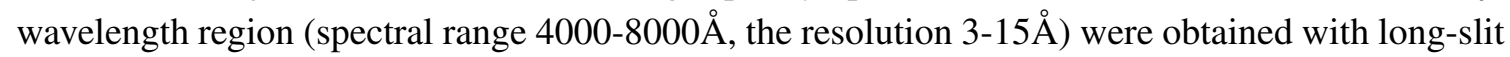
spectrographs equipped with a CCD. For details on data acquisitions, data reduction and calibration

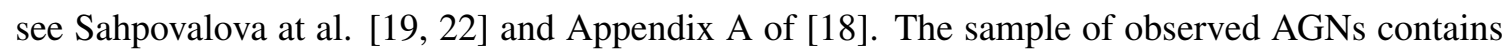
well-known and studied objects: NGC 5548, NGC 4151, 3C390.3, Ark 564, and Arp 102B. So far, 


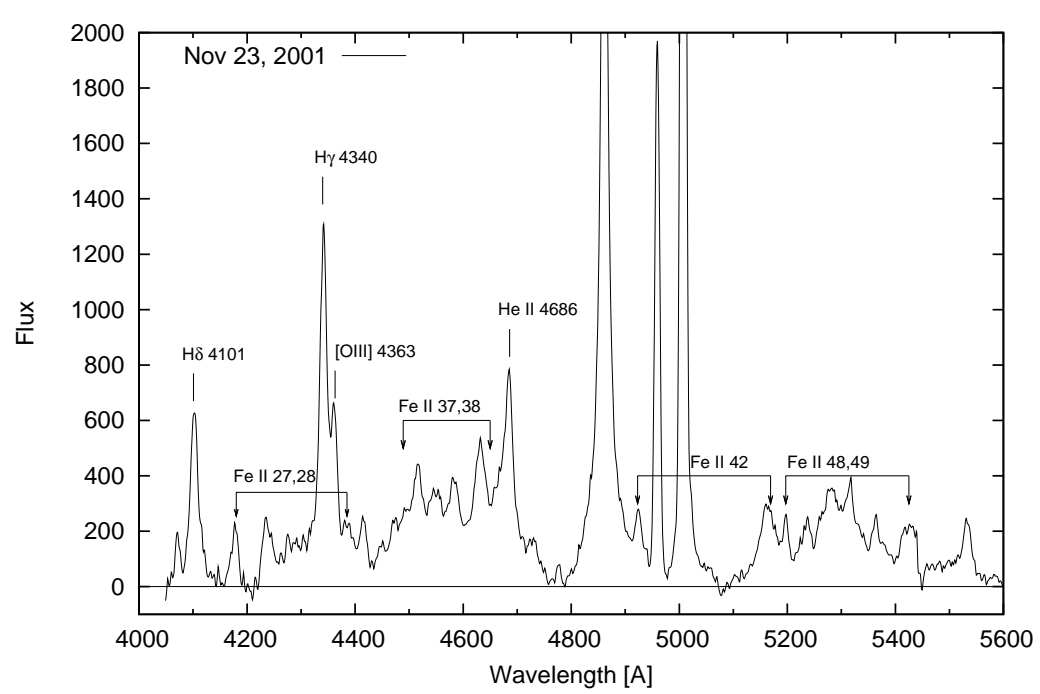

Figure 1: The Fe II emission around the $\mathrm{H} \beta$ line for the NLS1 galaxy Ark 564. Strong emission lines are labeled, as well as the Fe II multiplets [[23].

the results of this campaign have been presented and published in a number of papers (for more

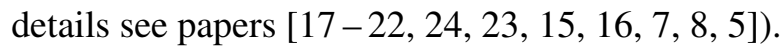

\section{The case of the NLS1 type AGN - Ark 564}

The observations of the NLS1 galaxy Ark 564 were performed from 1999 to 2010 (for details see [23]). An interesting feature of narrow-line Seyfert 1 galaxies is the prominent emission of Fe II in the optical band around the $\mathrm{H} \beta$ line (Fig. W), that is clearly visible in the spectra of these objects due to the relatively small width of the emission lines. From the multi-Gaussian fitting of the wavelength range of $4000-5600 \AA$, and the analysis of the Gaussian line widths and shifts, we have shown that the optical Fe II lines of this AGN are coming from the intermediate line region [D2]], as suggested before (see [ए]], and references therein).

During the monitoring period, the mean continuum and lines fluxes are weakly varying, as the decrease of only $20 \%-30 \%$ (see light curves in Fig. 5 in [23]) from the beginning (1999) to the end of the monitoring campaign (2010) was detected. Also, in the light curves, five flare-like events (two prominent and three possible) lasting $~ 1-3$ days were detected.

One result is particularly intriguing. It was observed, that there is almost no correlation between the $\mathrm{H} \alpha$ and $\mathrm{H} \beta$ line fluxes (Fig. ㄱ). Such behavior is unexpected if photoionization by the central continuum source would be the main mechanism responsible for the production of both lines. The lack of the correlation could indicate very complex physical processes in the line forming region, i.e. beside the photoionization some additional physical processes may be present [[23].

\section{The case of the DPL radio galaxy - $3 C 390.3$}

One of the monitored AGN is a radio galaxy 3C390.3 showing double-peaked broad emission lines. The observations of 3C 390.3 were performed from 1995 to 2007 . The results of these 


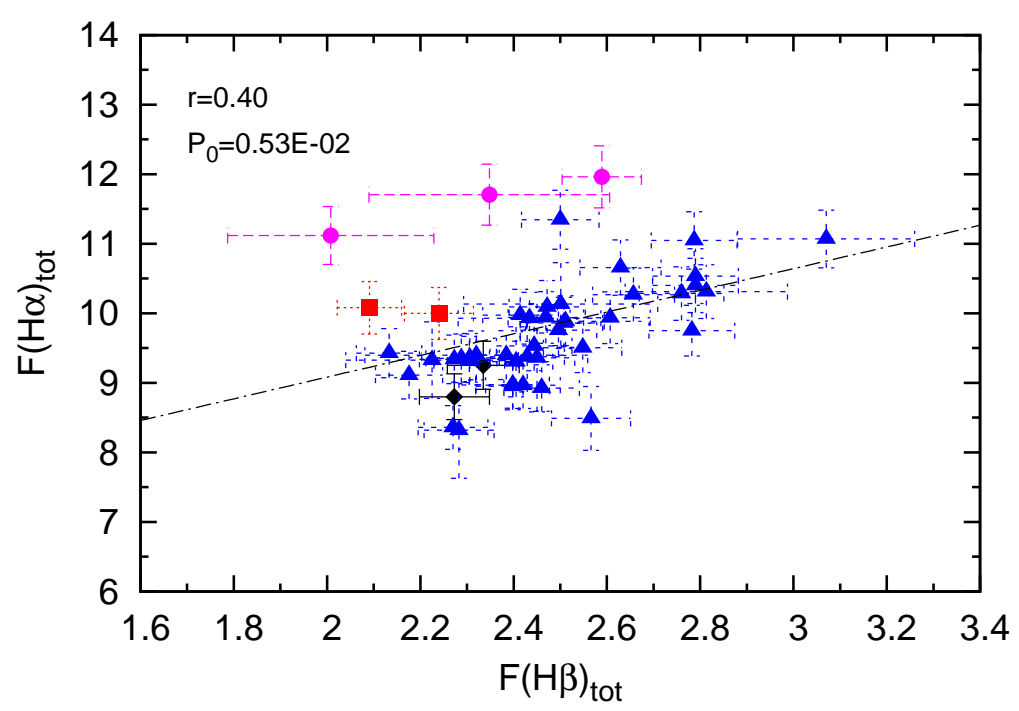

Figure 2: The $\mathrm{H} \alpha$ as a function of the $\mathrm{H} \beta$ line flux of Ark 564. The correlation coefficient and the corresponding P-value are given in the upper left corner. Data obtained with different telescopes are marked with different symbols: diamonds - $6 \mathrm{~m}$ in Russia, circles - $1 \mathrm{~m}$ Zeiss in Russia, triangles - 2.1m GHO in Mexico, squares - 2.1m SPM in Mexico [223].

investigations were published in [17], [22, [6, [8]. The double-peaked $\mathrm{H} \alpha$ and $\mathrm{H} \beta$ lines clearly indicate a disk-like geometry of the BLR. But, the variations in line profiles show that there is an additional emission component contributing to the line core, that is originating in the region different then the disk (see the mean and rms $\mathrm{H} \alpha$ spectra in Fig. B, and for more details see Fig. 12 in [22]. This additional component could be also explained as an extra emission coming from the perturbation in an accretion disk [ $[\mathbb{8}]$. The analysis of the line emission profiles from different epochs, i.e. the analysis of the line fluxes of different line segments has shown that the line-emitting part of the disc is shifting along the accretion disk from $R_{\text {inn }}=250 R_{\mathrm{g}}$ to $R_{\text {inn }}=550 R_{\mathrm{g}}$, during the first period of observations (until year 2002). After year 2002, the position of the emitting part of the disk is fixed (see for more details [ए]]).

\section{The case of the Seyfert 1.5 type AGN - NGC 4151}

This galaxy is a brightest Seyfert 1.5-type galaxy that has been studied in detail at all wavelengths by many authors. Latest result about this AGN has shown this object could actually host a supermassive binary black hole [5]. In our monitoring campaign, it was observed in period 1996-

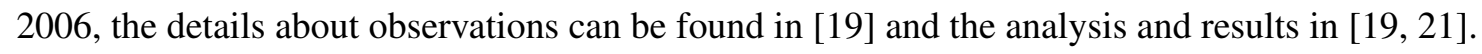

The continuum and line fluxes varied strongly (up to a factor 6) during the monitoring period. The emission was maximum in 1996-1998, and there were two minima, in 2001 and in 2005. As a consequence, the spectral type of the nucleus changed from a Seyfert 1.5 in the maximum activity to a Seyfert 1.8 in the minimum state (see Fig. 1 in [प्प]).

We emphasize here the behavior of the emission line fluxes with respect to the continuum flux. Fig. 团 shows the $\mathrm{H} \alpha$ and $\mathrm{H} \beta$ fluxes as a function of the continuum flux at $\lambda=5117 \AA$, 


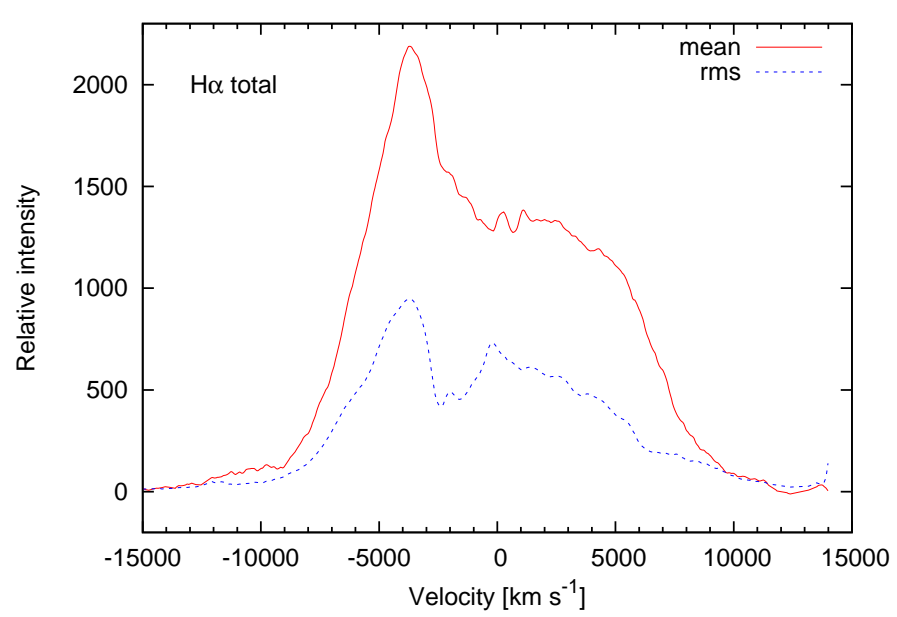

Figure 3: Mean and rms spectra from the whole monitoring period (1995-2007) of the $\mathrm{H} \alpha$ line of the DPL radio galaxy 3 C 390.3 [22]
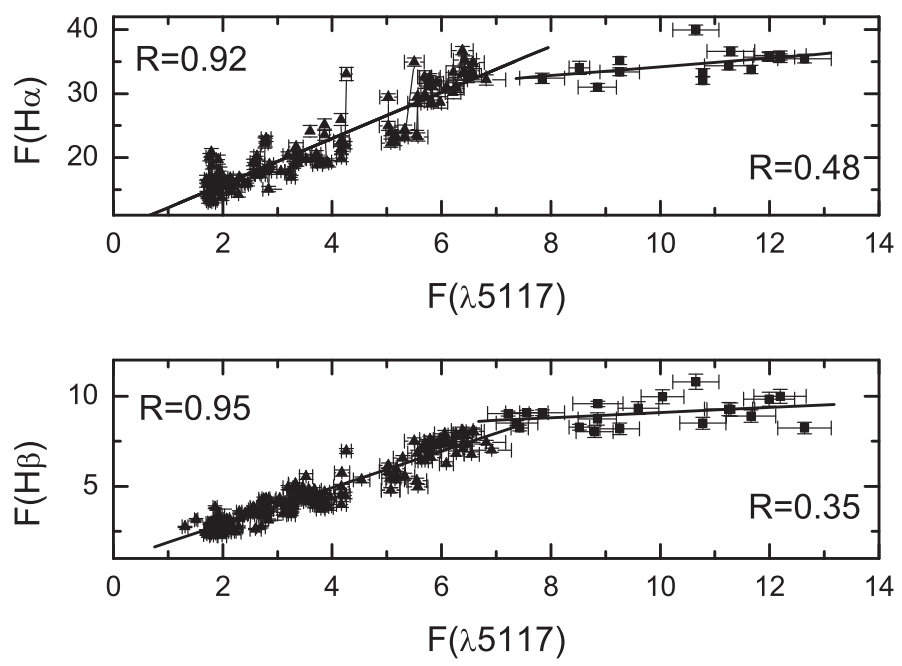

Figure 4: The $\mathrm{H} \alpha$ and $\mathrm{H} \beta$ line fluxes versus the continuum flux of Seyfert 1.5 galaxy NGC 4151 . The correlation coefficients $R$ are given for two sets of data. The line fluxes are given in units of $10^{-12} \mathrm{erg} \mathrm{cm}^{-2} \mathrm{~s}^{-1}$, and the continuum flux in $10^{-14} \mathrm{erg} \mathrm{cm}^{-2} \mathrm{~s}^{-1-1}$ [ए⿴囗口] .

where the correlation coefficients are calculated for two data subsets. When the continuum flux is small $\left(\mathrm{Fc} \leq 7 \cdot 10^{-14} \mathrm{erg} \mathrm{cm}^{-2} \mathrm{~s}^{-1-1}\right)$, there is a linear relationship between the emission line and the continuum flux variations (the correlation coefficient is high and above 0.9). This high correlation means that the ionizing continuum was a good extrapolation of the optical continuum. For a stronger continuum emission $\left(\mathrm{Fc} \geq 7 \cdot 10^{-14} \mathrm{erg} \mathrm{cm}^{-2} \mathrm{~s}^{-1-1}\right.$ ), the line fluxes are weakly correlated, and it seems that the lines are saturated for high continuum values (Fig. (A). The BLR of this object could consist from two regions, one being photoionized by the central continuum source and other a non-radiatively heated region [एव]. 


\section{Conclusions}

Here we have shortly presented the long-term spectral optical monitoring campaign of a sample of type 1 AGN. We have outlined just some important results related to representative of three type of objects (NLS1 Ark 564, DPL radio galaxy 3C390.3, Seyfert 1.5 NGC 4151) in the frame of the applicability of photoionization models and complex geometry and structure of the BLR. The geometry of the BLR is complex and usually there are contribution of more sub-regions with different geometry/motions to the total line profile. The BLR physics and line-formation mechanisms are complex, and probably other ionization mechanism, apart from the photoionization, are present. The objects presented here are special cases and might be unique, but nevertheless the complexity of the BLR should be taken into account when using the parameters of the BLR for the estimates of the mass of the supermassive black hole in the center of an AGN.

\section{References}

[1] Bahcall, J. N., Kozlovsky, B.-Z., Salpeter, E. E. 1972, ApJ 171467.

[2] Bentz, M. C., Denney, K. D., Cackett, E. M., et al. 2006, ApJ 651775.

[3] Bentz, M. C., Walsh, J. L., Barth, A. J., et al. 2010, ApJ 716993

[4] Blandford, R. D., McKee, C. F. 1982, ApJ 255419.

[5] Bon, E., Jovanović, P., Marziani, P., et al. 2012, ApJ 759118.

[6] Gaskell, C. M. 1988, ApJ 325114.

[7] Ilić, D. 2007, Ser. Astron. J. 17515.

[8] Jovanović, P., Popović, L. Č., Stalevski, M., Shapovalova, A. I. 2010, ApJ 718168.

[9] Kaspi, S., Smith, P. S., Netzer, H., et al. 2000, ApJ 533631.

[10] Kovačević, J., Popović, L. Č., Dimitrijević, M. S. 2010, ApJS 18915.

[11] Kollatschny, W., Dietrich, M. 1997, A\&A 3235.

[12] Peterson, B. M. 2008, New Astron. Rev. 52240.

[13] Peterson, B. M., Ferrarese, L., Gilbert, K. M., et al. 2004, ApJ 613682.

[14] Wanders, I., Peterson, B. M. 1996, ApJ 466174.

[15] Popović, L. Č., Shapovalova, A. I., Chavushyan, V. H., et al. 2008, PASJ 611.

[16] Popović, L. Č., Shapovalova, A. I., Ilić, D., et al. 2011, A\&A 528130.

[17] Shapovalova, A. I., Burenkov, A. N., Carrasco, L., et al. 2001, A\&A 376775.

[18] Shapovalova, A. I., Doroshenko, V T, Bochkarev, N. G., et al. 2004, A\&A 422925.

[19] Shapovalova, A. I., Popović, L. Č., Collin, S., et al. 2008, A\&A 486 99S.

[20] Shapovalova, A. I., Popović, L. Č., Bochkarev, N. G., et al. 2009, New Astronomy Review 53191.

[21] Shapovalova, A. I., Popović, L. Č., Burenkov, A. N., et al. 2010a, A\&A 509106.

[22] Shapovalova, A. I., Popović, L. Č., Burenkov, A. N., et al. 2010b, A\&A 51742.

[23] Shapovalova, A. I., Popović, L. Č., Burenkov, A. N., et al. 2012, ApJS 20210.

[24] Shapovalova, A. I., Popović, L. Č., Ilić, D., et al. 2011, Baltic Astronomy 20476. 